\title{
APLICAÇÃO DE UMA TEORIA TERMODINÂMICA NO ESTUDO DE UM VÓRTICE CICLÔNICO DE ALTOS NÍVEIS SOBRE O NORDESTE DO BRASIL
}

\author{
RAFAELA LISBOA COSTA ${ }^{1}$, ENIO PEREIRA DE SOUZA ${ }^{2}$, FABRICIO DANIEL DOS SANTOS \\ SILVA $^{3}$ \\ ${ }^{1}$ Universidade de Brasília, Instituto de Geociências, Brasília, DF, Brasil \\ ${ }^{2}$ Universidade Federal de Campina Grande, Unidade Acadêmica de Ciências Atmosféricas(UFCG/UACA), \\ Campina Grande, PB, Brasil. \\ ${ }^{3}$ Instituto Nacional de Meteorologia, Coordenação de Desenvolvimento e Pesquisa, (INMET/CDP), Brasília, \\ DF, Brasil. \\ rafaelalisboac@gmail.com, esouza@dca.ufcg.edu.br, fabricio.silva@inmet.gov.br
}

Recebido Novembro de 2012 - Aceito Junho de 2013

\begin{abstract}
RESUMO
Este estudo teve como objetivo principal aplicar a teoria da máquina térmica em um evento de Vórtice Ciclônico de Altos Níveis (VCAN), ocorrido sobre o Nordeste do Brasil com o intuito de analisar um dos mecanismos de manutenção desse tipo de sistema. A teoria da máquina térmica, baseada na segunda lei da termodinâmica, foi utilizada para calcular a diferença de pressão existente entre dois pontos localizados no centro e na periferia do VCAN. O modelo Brazilian developments on the Regional Atmospheric Modeling System (BRAMS) conseguiu representar bem o evento ocorrido no período de 10 a 21 de janeiro de 2011, mostrando uma maior intensidade deste evento no período de 16 a 19 de janeiro de 2011. A teoria da máquina térmica apresentou-se como uma ferramenta capaz de ser utilizada no estudo dos VCANs, mostrando boa concordância com as simulações realizadas com o modelo.
\end{abstract}

Palavras chave: Convecção Atmosférica, VCAN, Teoria da Máquina Térmica, BRAMS

\begin{abstract}
AN APPLICATION OF A THERMODYNAMIC THEORY ON STUDYING AN UPPER-TROPOSPHERIC CYCLONIC VORTEX OVER NORTHEAST BRAZIL

This study aimed to apply the heat engine theory to an event of Upper-Tropospheric Cyclonic Vortex (UTCV) occurred in Northeast Brazil in order to examine one of the mechanisms that maintains this type of system. The theory is based on the second law of thermodynamics and it was used to calculate the pressure difference between two points located at the center and periphery of UTCV. The Brazilian developments on the Regional Atmospheric Modeling System (BRAMS) model represented well the event occurred in the period of 10 to 21 January 2011, showing a higher intensity in this event from 16 to 19 January 2011. The heat engine theory showed as an useful tool to be used in the study of UTCVs, showing good agreement with the simulations performed with the model.
\end{abstract}

Keywords: Atmospheric Convection, UTVC, Heat-Engine Theory, BRAMS

\section{INTRODUÇÃO}

As circulações atmosféricas regionais e os sistemas sinóticos atuantes no Nordeste do Brasil (NEB) podem ter origem externa ou interna à região, e constituem os principais fatores dinâmicos que determinam a precipitação sazonal (Kayano e Andreoli, 2004). A precipitação nessa região depende do sistema atuante. Geralmente há má distribuição temporal, além de grande variabilidade espacial durante o ano (Costa, 2009). Os quatro principais sistemas meteorológicos causadores dessas intensas precipitações são: a Zona de Convergência Intertropical (ZCIT), que é responsável pelas precipitações que ocorrem no centro norte da região; os Sistemas Frontais que provocam chuva no centro sul, os Distúrbios Ondulatórios 
de Leste (DOLs) responsáveis pelas chuvas na costa leste do NEB e os Vórtices Ciclônicos de Altos Níveis (VCANs), que produzem chuvas, principalmente, na parte norte do NEB (Lima, 2009).

Durante o verão no Hemisfério Sul, meses de dezembro a fevereiro, a alta troposfera na América do Sul é caracterizada por uma circulação anticiclônica quase estacionária sobre a Bolívia, conhecida como Alta da Bolívia, e um cavado profundo localizado a leste da costa do NEB e sobre o Oceano Atlântico adjacente (Mishra et al., 2001; Newell et al., 1972). A costa leste do NEB é uma região de frequente ocorrência de distúrbios ciclônicos de escala sinótica durante os meses de verão (Satyamurty et al., 1998; Karoly e Vicent, 1998). Esses distúrbios são conhecidos como Vórtices Ciclônicos de Altos Níveis (VCANs), principais sistemas que influenciam diretamente o tempo na região tropical, especialmente o Nordeste do Brasil (NEB) e também em partes da região Sul e Sudeste do Brasil. Eles podem ser definidos como sistemas com núcleo de baixa pressão em escala sinótica, formados inicialmente na alta troposfera podendo se estender a níveis inferiores e cuja circulação ciclônica fechada possui o centro mais frio do que sua periferia (Costa, 2009; Kousky e Gan, 1981).

Amplamente estudados, os VCANs são classicamente conhecidos como sistemas ambíguos, pois, se por um lado são capazes de produzir grandes totais pluviométricos devido à atuação da convecção gerada ao longo de sua periferia, por outro, podem inibir drasticamente a formação de nuvens devido ao movimento descendente de ar frio e seco no seu centro (Costa, 2009). Esse potencial em influenciar fortemente o regime pluviométrico de uma localidade em um curto espaço de tempo faz com que ele seja classificado como um sistema extremamente importante, independente da região em que atue.

No intuito de melhor compreender os vários sistemas meteorológicos atuantes por todo o globo, mais precisamente os que atuam no Brasil, vários métodos são utilizados. A utilização de modelos atmosféricos tem sido aceita como uma das ferramentas importantes na análise e previsão dos vários eventos meteorológicos ocorrentes. Além da utilização dos modelos numéricos atmosféricos, o uso de teorias capazes de analisar esses eventos meteorológicos é uma prática bastante difundida.

Utilizada em vários estudos, como de Emanuel (1986) e Rennó e Ingersoll (1996), a teoria da máquina térmica, baseada no princípio da convecção atmosférica como uma máquina de Carnot, é bastante utilizada a fim de se compreender sobre os mecanismos de formação desses sistemas. Com o uso desta teoria, foi possível explicar a circulação geral da atmosfera (Lorenz, 1967), a convecção atmosférica natural, sistemas como furacões e trombas d'água (Rennó e Ingersol, 1996; Rennó et al. 1998 e Rennó e Bluestein, 2001), assim como, a formação de circulações termodinamicamente forçadas em superfícies heterogêneas (Souza, 2004; Alcântara e Souza, 2009).

Uma máquina térmica é definida como um mecanismo que transforma calor em trabalho. Segundo Woolard (1926), a atmosfera age como uma máquina térmica gigante, transformando energia radiante do Sol em energia para "alimentação" dos fenômenos meteorológicos. De acordo com o autor, o reservatório quente da máquina térmica atmosférica seriam as áreas do planeta que recebem mais energia do Sol e, consequentemente, se aquecem mais, como os trópicos; já o reservatório frio seria aquelas regiões bem mais frias do planeta, onde há perda de calor, ou seja, os pólos.

A convecção atmosférica, resultado, também, da atuação dos vários sistemas meteorológicos, pode ser descrita utilizando a teoria da máquina térmica. Durante um ciclo da convecção como uma máquina térmica, calor é retirado da camada superficial (fonte de calor) e uma porção dele é rejeitada para a troposfera livre (sumidouro de calor) de onde é irradiado para o espaço. O balanço é transformado em trabalho mecânico que é gasto na manutenção dos movimentos convectivos contra a dissipação mecânica.

Desta forma, tendo em vista as várias aplicações desta teoria, o presente trabalho teve por objetivo principal aplicar a teoria da máquina térmica na análise de um evento de VCAN sobre a região Nordeste do Brasil (NEB), com o intuito de se compreender o mecanismo termodinâmico de manutenção desses sistemas meteorológicos e verificar se essa teoria é capaz de explicar parte da manutenção termodinâmica desse sistema.

\section{MATERIAIS E METODOLOGIA}

\subsection{Teoria da máquina térmica}

A teoria termodinâmica, descrita em detalhes por Souza e Rennó (2000), combina uma equação de energia para uma parcela em convecção com a primeira e a segunda leis da termodinâmica. O objetivo é obter uma equação para a queda de pressão associada a uma circulação convectiva entre o seu ponto de alta pressão (ponto A) e o seu ponto de baixa pressão (ponto B). Para melhor se entender a contribuição da inclinação do terreno nos processos estudados, Souza e Rennó (2000) e Souza (2004) dividiram a diferença de temperatura entre A e B em duas partes, uma adiabática e outra não-adiabática. A equação final para a queda de pressão fica:

$$
\begin{aligned}
& \Delta \mathrm{p} \approx \mathrm{p}_{\mathrm{A}}\left\{1-\exp \left[\frac{\gamma \eta}{(\gamma \eta-1) \mathrm{R}}\left(\frac{\mathrm{c}_{\mathrm{p}} \Delta \mathrm{T}_{\mathrm{na}}}{\overline{\mathrm{T}_{\mathrm{S}}}}+\frac{\mathrm{L}_{\mathrm{v}} \Delta \mathrm{r}}{\overline{\mathrm{T}_{\mathrm{S}}}}\right)-\frac{\Delta \mathrm{z}}{\mathrm{H}_{\mathrm{S}}}\right]\right\} \\
& \Delta T=\Delta T_{a d}+\Delta T_{n a}=-\frac{g}{c_{p}} \Delta z+\Delta T_{n a} \Rightarrow \Delta T_{n a}=T_{B}-T_{A}+\frac{g}{c_{p}} \Delta z
\end{aligned}
$$


em que $\eta$ é a eficiência termodinâmica, $\gamma$ é a fração da dissipação total, que ocorre na região de absorção de calor nas proximidades da superfície, $\Delta p=p_{A}-P_{B}$ é a diferença de pressão entre dois pontos A e B sobre cada superfície, $\Delta r=r_{A}-r_{B}$ é a variação da razão de mistura, $\Delta \boldsymbol{z}=\boldsymbol{z}_{A}-\boldsymbol{z}_{B}$ é a diferença de altitude, $\overline{\mathrm{T}}_{\mathrm{S}}$ é a temperatura média do ar próximo a superfície entre os pontos A e B, $H_{S} \equiv \frac{R \bar{T}_{S}}{g}$ é a altura de escala, $\mathrm{c}_{\mathrm{P}}$ é o calor especifico à pressão constante e $\mathrm{L}_{\mathrm{v}}$ é o calor latente de vaporização.

Os resultados da aplicação da teoria para a queda de pressão em brisas foram apresentados nos estudos de por Souza (2004) e Alcântara e Souza (2009). Nesses estudos, a parte que envolve a liberação de calor latente foi desprezada, por significar um efeito menor. Entretanto, para vórtices saturados, o termo envolvendo a variação de razão de mistura tem um papel importante (Rennó e Bluestein, 2001; Rennó, 2008).

Derivando-se logaritmicamente a equação que define a temperatura potencial equivalente de saturação, tem-se que:

$$
d\left(\ln \theta_{\text {es }}\right)=d(\ln \theta)+d\left(\frac{L_{v} r_{s}}{c_{p} T}\right)
$$

Lembrando que a diferença de pressão não hidrostática será avaliada no nível médio de $1000 \mathrm{hPa}$, pode-se considerar: $\theta=\mathrm{T}_{\mathrm{s}} ; \mathrm{e} \mathrm{d}\left(\frac{\mathrm{r}}{\mathrm{T}}\right) \approx \frac{1}{\mathrm{~T}} \mathrm{dr}$ (na realidade, isso desfaz a simplificação realizada na derivação da temperatura potencial equivalente). A partir dessas considerações, com $\mathrm{T}=\mathrm{T}_{\mathrm{s}}$, tem-se:

$$
\mathrm{c}_{\mathrm{p}} \mathrm{d} \ln \theta_{\mathrm{es}}=\mathrm{c}_{\mathrm{p}} \frac{\mathrm{dT}}{\mathrm{T}_{\mathrm{s}}}+\frac{\mathrm{L}_{\mathrm{v}} \mathrm{dr}_{\mathrm{s}}}{\mathrm{T}_{\mathrm{s}}}
$$

Como foi considerada a pressão reduzida ao nível médio do mar, tem-se que $\Delta \mathrm{z}=0$. Logo, a Equação 1 pode ser reescrita da seguinte forma:

$$
\Delta \mathrm{p} \approx \mathrm{p}_{\mathrm{A}}\left\{1-\exp \left[\frac{\gamma \eta}{(\gamma \eta-1) \mathrm{R}} \mathrm{c}_{\mathrm{p}} \Delta \ln \theta_{\mathrm{es}}\right]\right\}
$$

A Figura 1 mostra, esquematicamente, o comportamento da convecção atmosférica observada na borda de um VCAN como uma máquina térmica. $\mathrm{O}$ ponto " $\mathrm{A}$ " representa o raio de influência do vórtice, ou seja, o raio de uma curva envolvente da região onde o fluxo converge para o vórtice. O ponto " $\mathrm{B}$ " representa um ponto ao longo da trajetória espiral da parcela de ar em torno do vórtice na região do vento tangencial máximo, onde os ventos convergem. O ponto " $\mathrm{C}$ " representa o ponto de estagnação, onde os ventos horizontais se anulam, perto da superfície no centro do vórtice. Finalmente, o ponto " $D$ " representa a região de rejeição de calor (divergência dos ventos), de acordo com a nomenclatura de Rennó (2008).

\subsection{Descrição da simulação}

Para este estudo, fez-se uma simulação utilizando o

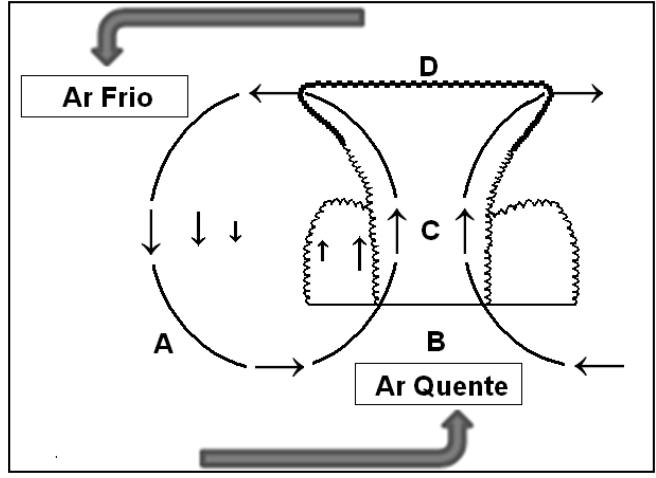

Figura 1 - Esquema de um VCAN como uma máquina térmica natural.

modelo BRAMS, na versão 4.2 descrita por Freitas et al. (2007), com grade de $20 \mathrm{~km}$ de resolução, para um evento de VCAN ocorrido sobre a região NEB em Janeiro de 2011, causador de intensa precipitação sobre alguns estados. Para tanto, utilizou-se na simulação o esquema de parametrização de Grell (Grell e Dévényi, 2002). A simulação possuiu as seguintes características básicas: o modelo BRAMS inicializado a 00:00 UTC do dia 10 de janeiro de 2011 e integrado durante um período de 120 horas. Foram utilizados dados diários de reanálises do National Center for Atmospheric Research/ National Centers for Environmental Predictions (NCAR/ NCEP), das variáveis temperatura do ar, altura geopotencial, componentes zonal e meridional do vento, pressão atmosférica e razão de mistura no nível de $200 \mathrm{hPa}$, na inicialização do modelo, na análise sinótica do período e no cálculo da teoria da maquina térmica.

Para a realização da análise sinótica, além do uso dos dados de reanálise, foram utilizadas imagens do satélite GOES12 , no canal infravermelho, para o período de 10 a 21 de janeiro, disponíveis na pagina do Centro de Previsão do Tempo e Estudos Climáticos/ Instituto Nacional de Pesquisas Espaciais (CPTEC/ INPE), pelo endereço: http://satelite.cptec.inpe.br/home.

Juntamente com o modelo BRAMS, usado primeiramente para identificar o VCAN, as equações da teoria termodinâmica foram aplicadas para calcular a diferença de pressão em duas regiões distintas do sistema $\mathrm{N}$, localizadas em seu centro (Ponto A) e sua periferia (Ponto B). A Figura 2 mostra a localização aproximada destes pontos.

Para o caso estudado, aplicou-se a teoria da máquina térmica para melhor compreender a diferença de pressão existente entre o centro do VCAN e sua periferia. Para tanto, a eficiência termodinâmica considerada foi de $10 \%$, ou seja, $\eta$ $=0,1$ e o parâmetro $\gamma$ foi considerado igual a 1. Essas mesmas considerações foram feitas por Rennó (2008), pois esses são os valores consagrados na literatura para sistemas precipitantes associados à convecção profunda. 


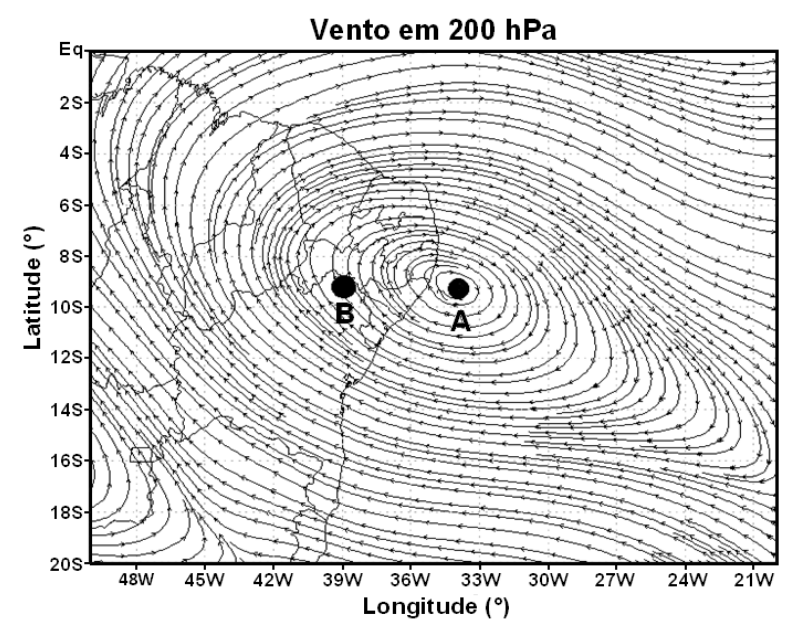

Figura 2 - Localização dos pontos analisados no estudo: centro (ponto A) e periferia (ponto B) do VCAN para o dia 16 de janeiro de 2011 , gerado pelo modelo BRAMS.

\subsection{Análise estatística}

Neste estudo, foram realizadas as devidas comparações entre a simulação realizada com o modelo e a aplicação da teoria da máquina térmica, a fim de analisar se a diferença da pressão entre a periferia e o centro do VCAN pode ser avaliada através da teoria termodinâmica. Para tanto, utilizaram-se quatro índices estatísticos: o Erro Absoluto Médio, Erro Quadrático Médio, o Coeficiente de Correlação de Pearson e o Coeficiente de Determinação, através da análise de regressão linear.

\section{RESULTADOS E DISCUSSÕES}

\subsection{Caracterização do ambiente sinótico}

A Figura 3 mostra as linhas de corrente, obtidas através dos dados de reanálises, no nível de $200 \mathrm{hPa}$, para a América do Sul (Figura 3b) e para o Nordeste do Brasil (Figura 3a), e a imagem de satélite para o dia 16 de janeiro (Figura 3c) para a análise do evento, ocorrido entre os dias 10 a 21 de janeiro de 2011. Entre os dias 16 a 20 de janeiro, esse sistema apresentou-se mais intenso e deslocou-se para o interior do continente. É possível observar uma circulação ciclônica fechada localizada entre o Oceano Atlântico e a costa leste do NEB. O centro do vórtice está posicionado sobre o Oceano Atlântico, próximo ao litoral sul de Pernambuco e litoral norte de Alagoas (Figuras 3a e 3b). O padrão anticiclônico da Alta da Bolívia (AB) aparece com uma configuração intensa, deslocada para o Oceano Pacífico (Figura 3b). Este VCAN não apresentou uma configuração clássica (Kousky e Gan, 1981) e sim, a configuração associada com uma circulação anticiclônica ao sul do VCAN, que se localizou na região do sudeste e oceano adjacente (Ramírez et al, 1999; Ferreira et al, 2004). Na imagem de satélite é possível analisar a atividade convectiva associada à região da periferia do VCAN. Aparentemente, a atividade convectiva apresenta-se com uma configuração amena, com algumas áreas onde se observa convecção profunda isolada.

\subsection{Aplicação da teoria da máquina térmica}

Nesta seção analisa-se e compara-se a simulação realizada com o modelo e a teoria para a diferença de pressão observada entre o centro (ponto A) e a periferia (ponto B) do VCAN, obtidas das simulações (DeltaP simulada) e calculadas através da teoria (DeltaP calculada) (Figuras 4 e 5) para o experimento em questão. Como já mencionado, para a aplicação da teoria da máquina térmica no estudo do VCAN, considerou-se

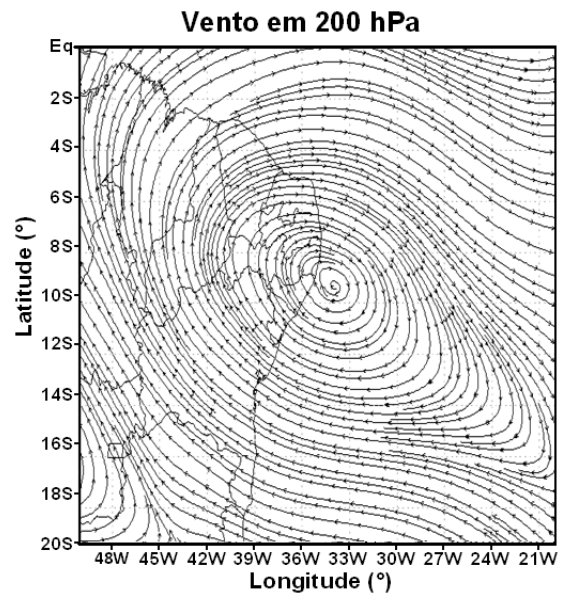

(a)

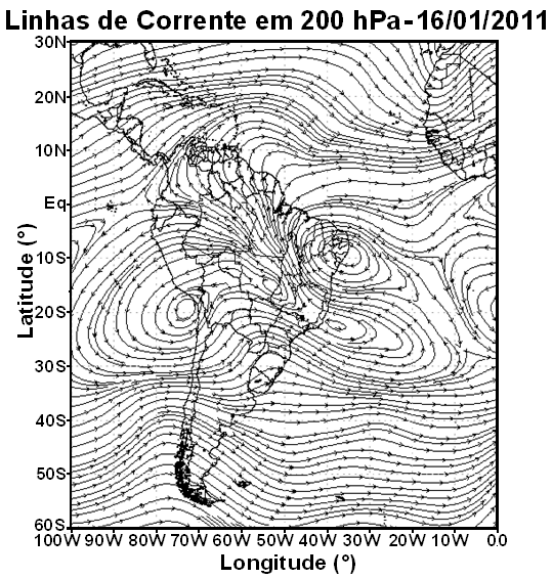

(b)

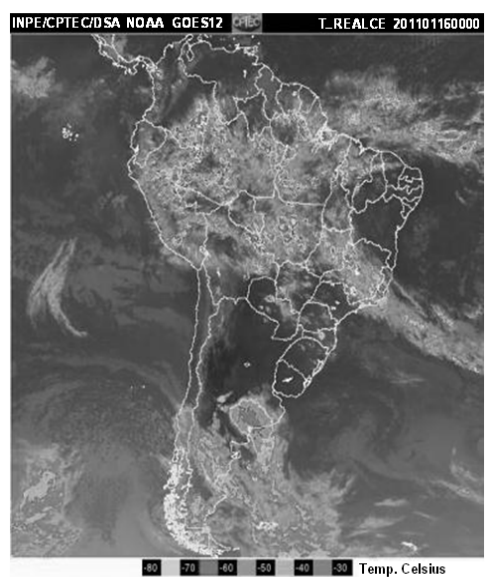

(c)

Figura 3 - Linhas de corrente em 200 hPa simulada, pelo BRAMS, para: (a) o Nordeste, (b) a América do Sul e (c) imagem de satélite às 00h00 UTC no dia 16 de janeiro. 

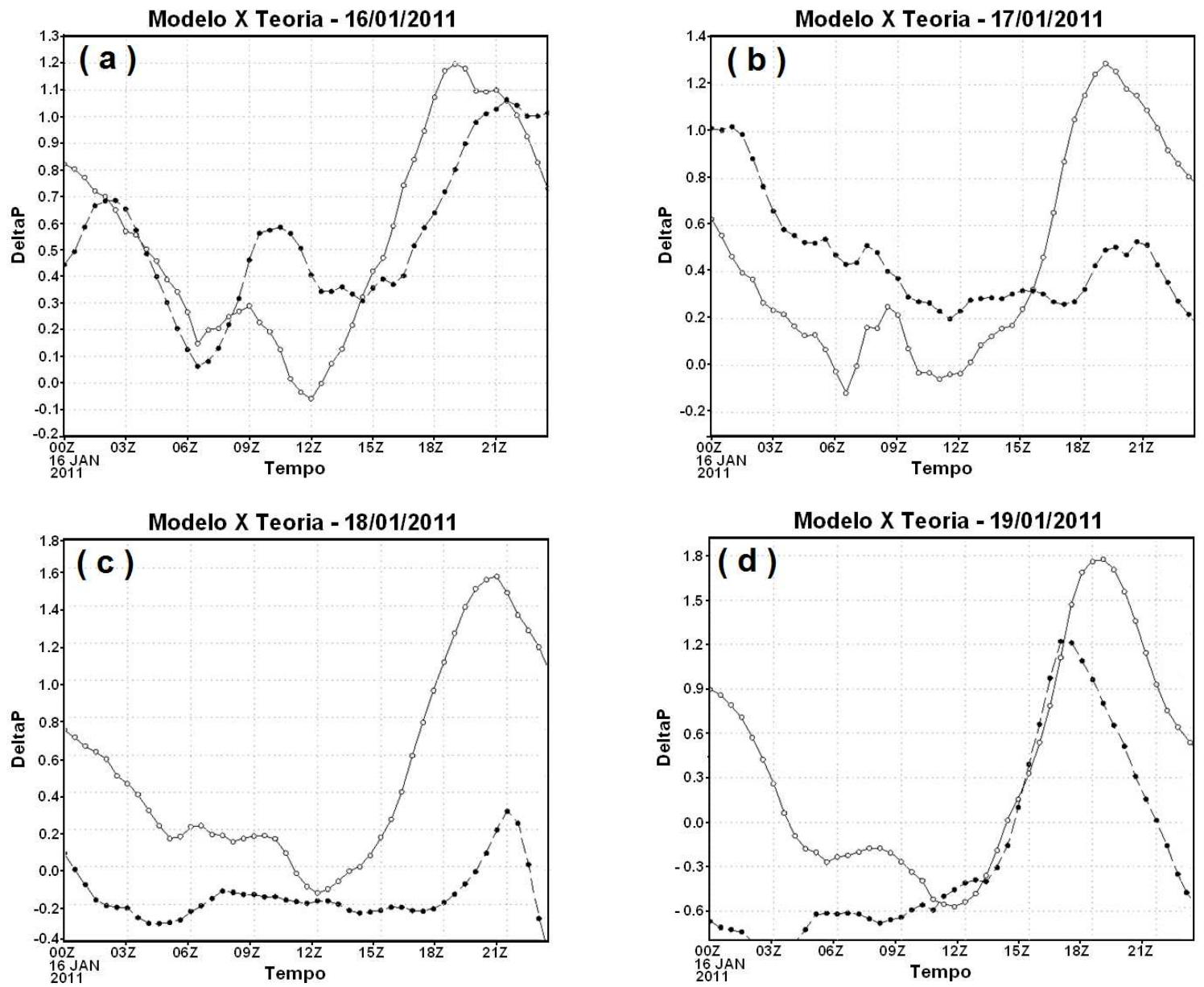

Figura 4 - Diferença de pressão obtida através das simulações (DeltaP simulada) (círculos brancos) e calculada através da teoria (DeltaP calculada) (círculos pretos) para os dias: 16 (a), 17 (b), 18 (c) e 19 (d) de janeiro de 2011.

uma eficiência termodinâmica de $10 \%$, como proposto por Rennó (2008) para convecção profunda.

$\mathrm{Na}$ Figura 4, para o dia 16 de janeiro (Figura 4a), é observado que o modelo mostra uma diferença de pressão mais intensa e positiva por volta das $18 \mathrm{UTC}, 15 \mathrm{~h} 00$ horário local. Isso ocorre devido à interação desse sistema com a superfície, que se apresenta mais aquecida e, sendo assim, é onde a convecção atmosférica torna-se mais intensa durante a tarde. Calculando essa queda da pressão com a teoria termodinâmica, observa-se que a curva tende a se comportar de forma semelhante ao modelo, apresentando apenas uma discrepância por volta das 09 UTC, 06h00 horário local. Para o dia 17 de janeiro (Figura 4b), observou-se que a teoria mostrou uma diferença de pressão mais acentuada do que o modelo, superestimando os resultados da simulação. Por volta do período da tarde, há uma inversão, com o modelo simulando uma queda acentuada da pressão entre a periferia e o centro do VCAN. A teoria também mostra essa diferença, entretanto, não tão acentuada quanto à simulada pelo modelo. Já para o dia 18 de janeiro (Figura 4c), há uma concordância entre a simulação do modelo e a teoria, porém, o modelo continua simulando valores mais elevados do que a teoria termodinâmica. $\mathrm{O}$ que pode ser observado no dia 19 de janeiro (Figura 4d) é um comportamento das curvas semelhante ao observado no dia 16 (Figura 4a). Mesmo sendo observada uma queda da pressão acentuada pelo modelo, a diferença da pressão calculada através da teoria mostra uma melhor aproximação com os valores apresentados pelo modelo.

A Figura 5, de forma geral, mostra o comportamento da diferença da pressão em todos os dias analisados, para as coordenadas de $9^{\circ} \mathrm{S}$ e $37^{\circ} \mathrm{W}$. Percebe-se que a teoria seguiu a tendência da simulação com o modelo, sendo observada uma concordância entre ambos.

Para uma melhor compreensão e como uma forma de quantificar a relação existente entre as simulações feitas com o modelo e a teoria termodinâmica, são apresentados os coeficientes de correlação, e seus respectivos gráficos de dispersão. A Figura 6 apresenta as correlações entre a diferença de pressão simulada pelo modelo e a calculada pela teoria. 


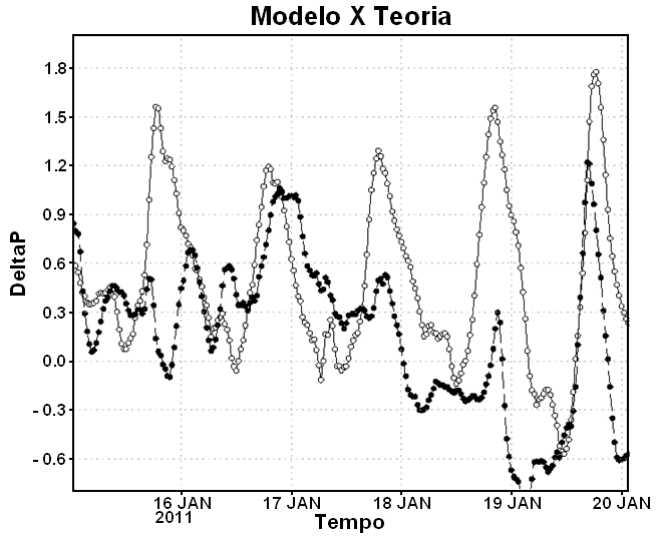

Figura 5 - Diferença de pressão obtida através das simulações (DeltaP simulada) (círculos brancos) e calculada através da teoria (DeltaP calculada) (círculos pretos).

São apresentadas as equações da reta de regressão linear correspondentes e os coeficientes de determinação $\left(r^{2}\right)$ e de correlação (r).

Os coeficientes de correlação obtidos, em relação ao período analisado foram: para o dia $16 / 01 \mathrm{r}=0,73$, o que indica uma correlação forte; para o dia 17/01 $\mathrm{r}=0,07$, o que implica em correlação bem fraca; para o dia 18/01 r =0,10, o que indica uma correlação bem fraca; para o dia 19/01 r = 0,70, o que implica em correlação forte. Os fracos valores de correlações observadas nos dias 17 e 18 ocorreram devido ao enfraquecimento do VCAN neste período, e subsequentemente a um fortalecimento do mesmo por volta do dia 19, como já

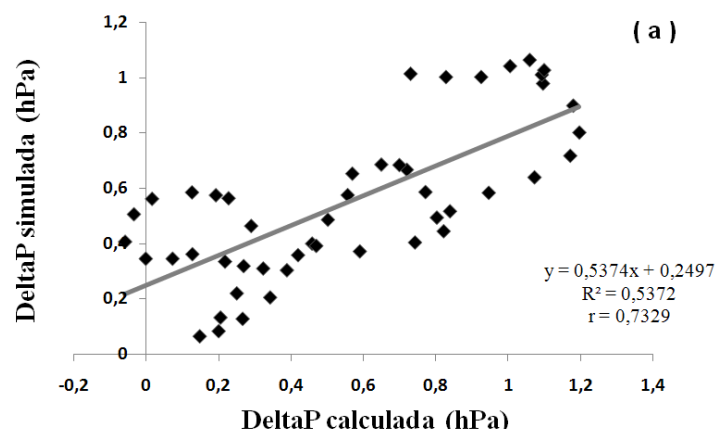

(c)

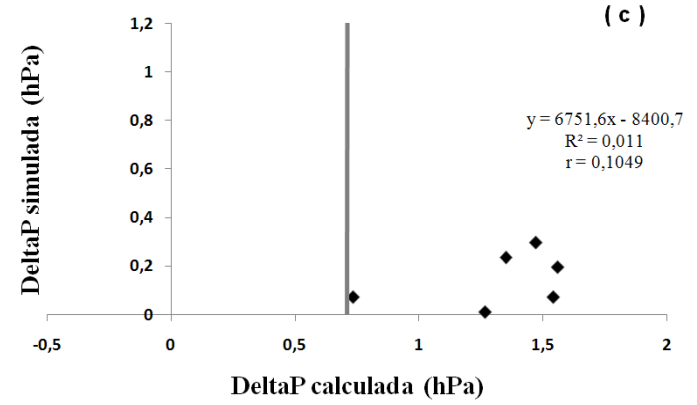

visto anteriormente. Portanto, no geral, os maiores valores do coeficiente de determinação foram verificados nos dias 16 e 19 .

A Tabela 1 mostra, resumidamente, os coeficientes de determinação $\left(\mathrm{r}^{2}\right)$, de correlação $(\mathrm{r})$, os erros absolutos (EA) e os erros quadráticos médios (EQM), obtidos neste estudo. Percebe-se o melhor desempenho do modelo nos dias 16 e 19. No dia 19, a correlação é alta e o valor do erro absoluto é alto devido às maiores diferenças entre os valores simulados $\mathrm{e}$ observados no período da tarde e da noite, embora as curvas se mostrem muito coincidentes.

A Figura 7 sintetiza todo o período de simulação do modelo versus o cálculo teórico da diferença de pressão. Apesar do coeficiente de determinação explicar aproximadamente $20 \%$ da variabilidade entre os dados, o coeficiente de correlação para todo o período foi de $r=0,44$, um valor que indica uma correlação moderada entre os valores simulados e calculados pela teoria. De acordo com o teste t-Student, para quatro dias de simulações com saídas a cada 30 minutos, entre 16 e 19 de janeiro de 2011, tem-se $\mathrm{N}=192$, e a probabilidade obtida para que se aceite a hipótese de dados não correlacionados foi $<0,0001$, o que mostra que se deve rejeitar a hipótese de que não existe correlação entre os dados. Logo, mesmo não sendo uma correlação forte, ainda assim, a teoria da máquina térmica pode ser aceita para se analisar a diferença da pressão entre dois pontos, no caso do VCAN, entre a periferia e o centro.

Tal análise mostra que embora existam, durante o período estudado, dias com coeficientes de correlação e determinação baixos, isso se dá em virtude de estes índices serem muito

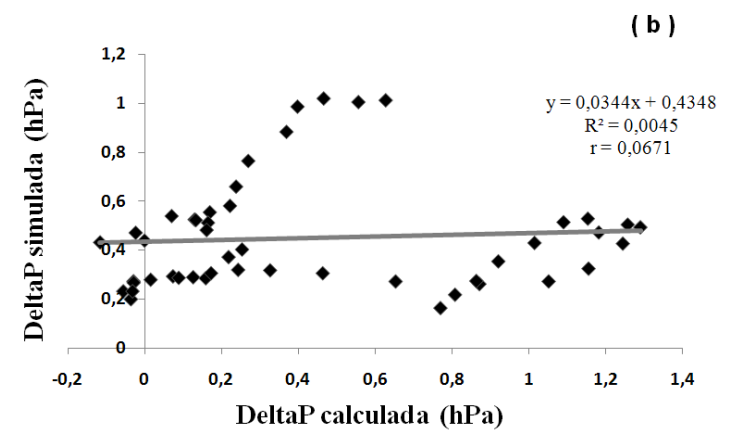

(d)

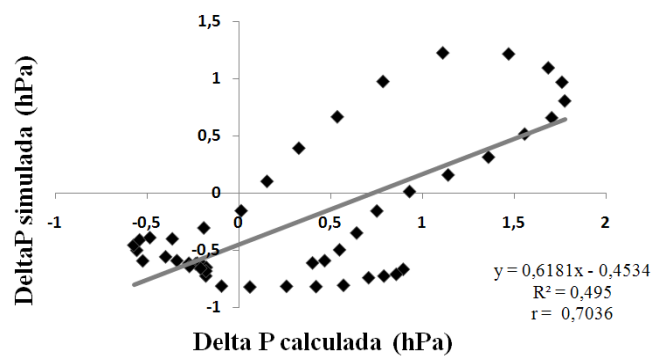

Figura 6 - Linha de tendência e correlação entre a diferença de pressão calculada através da teoria (DeltaP calculada) e a diferença de pressão obtida nas simulações (DeltaP simulada) para os dias: 16 (a), 17 (b), 18 (c) e 19 (d) de janeiro de 2011. 
Tabela 1 - Índices estatísticos analisados: Coeficiente de determinação $\left(\mathrm{R}^{2}\right)$, coeficiente de correlação (r), erro absoluto (EA) e erro quadrático médio (EQM).

\begin{tabular}{ccccc}
\hline Dia / Índices & $\mathbf{R}^{\mathbf{2}}$ & $\mathbf{r}$ & $\mathbf{E A}$ & EQM \\
\hline $\mathbf{1 6 / 0 1}$ & $\mathbf{0 , 5 3 7 2}$ & $\mathbf{0 , 7 3 2 9}$ & $\mathbf{0 , 1 9 8 9}$ & $\mathbf{0 , 2 5 4 3}$ \\
$\mathbf{1 7 / 0 1}$ & 0,0045 & 0,0671 & 0,4261 & 0,4744 \\
$\mathbf{1 8 / 0 1}$ & 0,011 & 0,1049 & 0,6948 & 0,8354 \\
$\mathbf{1 9 / 0 1}$ & $\mathbf{0 , 4 9 5}$ & $\mathbf{0 , 7 0 3 6}$ & $\mathbf{0 , 6 2 8 9}$ & $\mathbf{0 , 7 8 5 5}$ \\
\hline
\end{tabular}

sensíveis a valores extremos, portanto o modelo pode "simular" bem na maior parte das vezes. Contudo, se não conseguir capturar a tendência de valores observados muito altos ou muito baixos (neste caso a diferença de pressão simulada e calculada), o coeficiente de correlação apresentará valor muito baixo ou até mesmo negativo, como aconteceu para a análise do dia 15 de janeiro e que não foi considerado neste estudo. Consequentemente o valor de $\mathrm{r}^{2}$ será ainda mais reduzido. De maneira contrária, um modelo ruim poderá apresentar bons valores de $r$ e $r^{2}$ se conseguir captar algum valor extremo.

A Tabela 2 mostra os valores dos parâmetros obtidos para o teste t-Student, para os dias 16 a 19 individualmente, e para a série de todo o período entre os dias 16 e 19 de janeiro de 2011.

$\mathrm{Na}$ tabela, os $\mathrm{r}^{*}$ foram estatisticamente significativos a um nível acima de $99 \%$. Os dos dias 17 e 18 não foram significativos, pois deveriam apresentar valores de $\mathrm{r}$ acima de 0,53. O período como um todo, entre 16 e 19 de janeiro, apresentou alta significância estatística para o valor de $\mathrm{r}$ obtido, 0,44, superando um valor crítico para $r$ que seria de 0,26, comprovando a teoria de que as simulações e os valores calculados apresentam padrões condizentes entre si, sendo bem correlacionados.

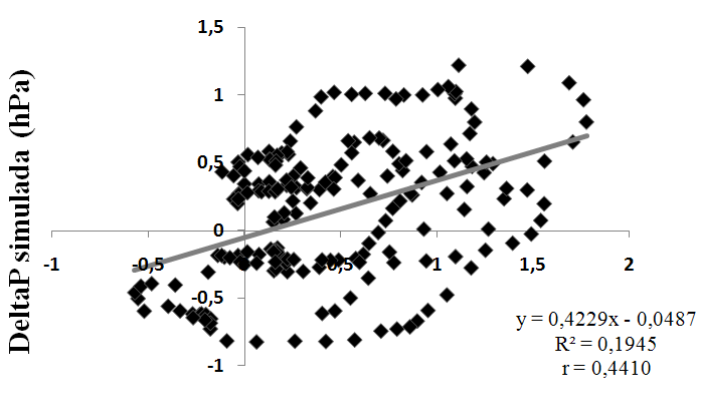

DeltaP calculada (hPa)

Figura 7 - Linha de tendência e correlação entre a diferença de pressão calculada através da teoria (DeltaP calculada) e a diferença de pressão obtida nas simulações (DeltaP simulada) para o período de 16 a 19 de janeiro de 2011.

\section{CONCLUSÕES}

Observou-se uma boa representação, pelo modelo atmosférico BRAMS, do evento de VCAN ocorrido sobre a região NEB, no período de 10 a 21 de janeiro de 2011.

Foi apresentada neste trabalho uma simples aplicação de uma teoria termodinâmica, que permitiu analisar a atividade convectiva associada ao VCAN e calcular a queda de pressão que ocorre entre o centro e a periferia desse sistema. A teoria da máquina térmica foi aplicada neste trabalho para avaliar de forma mais simples a diferença de pressão não-hidrostática entre dois pontos e, assim, estudar as características termodinâmicas em áreas distintas sob a atuação de um VCAN, mais precisamente, na região da periferia do sistema onde ocorrem os processos convectivos e o centro do sistema, caracterizado pela inibição da convecção.

Os valores da diferença de pressão entre os dois pontos fixos calculados teoricamente e simulados pelo modelo, entre os dias 16 e 19 de janeiro, mostraram a habilidade do modelo em representar esta variável. O modelo conseguiu capturar bem a variabilidade diurna da pressão não-hidrostática, principalmente no período da tarde, quando os processos de convecção geralmente são iniciados.

Entre os dias analisados, dois destes, em especial, apresentaram correlações altas, 16 e 19 de janeiro, e dois apresentaram baixas correlações, 17 e 18. Isso está associado ao fato de que a área da periferia e do centro do VCAN estava sobre os pontos fixados nos dias 16 e 19, e assim a teoria termodinâmica pôde ser aplicada com mais sucesso por ser mais dependente de processos convectivos, que ajudam na maior variação diurna da pressão atmosférica, principalmente entre os períodos da manhã e tarde. Nos dias 17 e 18 o deslocamento do VCAN deixou praticamente apenas a área do seu centro sobre estes pontos, esta região de subsidência, estabilidade e de pequena variação de pressão, influenciou no cálculo teórico, mostrando pouca variação diurna não acompanhando de forma satisfatória a simulação do modelo.

Ao analisar toda a variabilidade entre teoria e simulação para os 4 dias consecutivos, naturalmente, o coeficiente de 
Tabela 2 - Parâmetros obtidos através do teste t-Student para os dias analisados.

\begin{tabular}{cccccc}
\hline Parâmetros/Dia & $\mathbf{1 6 / 0 1}$ & $\mathbf{1 7 / 0 1}$ & $\mathbf{1 8 / 0 1}$ & $\mathbf{1 9 / 0 1}$ & $\mathbf{1 6}$ a 19/01/2011 \\
\hline $\mathbf{T}$ & 7,306 & 0,456 & 0,715 & 6,716 & 6,773 \\
$\mathbf{N}$ & 48 & 48 & 48 & 48 & 192 \\
$\mathbf{R}$ & $\mathbf{0 , 7 3 2 9 *}$ & 0,0671 & 0,1049 & $\mathbf{0 , 7 0 3 6 *}$ & $\mathbf{0 , 4 4 1 0 *}$ \\
\hline
\end{tabular}

correlação entre simulação e teoria não foi tão alto quanto nos dias 16 e 19, nem tão baixo quanto nos dias 17 e 18, mas ainda assim apresentou um valor de 0,44 , considerado moderado pela literatura. Embora visualmente as curvas teórica e simulada tenham mostrado forte coerência entre si, foi aplicado o teste t-Student para verificar a significância estatística da correlação a fim de testar a veracidade desta conexão entre as curvas teórica e simulada.

O teste mostrou que apesar do coeficiente de correlação ter-se mostrado moderado e não muito elevado, este foi estatisticamente significativa a um nível de $99 \%$, podendo-se rejeitar a hipótese de que os dados não poderiam estar correlacionados. Desta forma, comprovou-se que o uso da teoria foi bem sucedido, tendo-se que verificar bem quais os pontos onde esta será aplicada.

\section{AGRADECIMENTOS}

O primeiro autor agradece à Coordenação de Aperfeiçoamento de Pessoal de Nível Superior (Capes) pela concessão de bolsa durante o curso de mestrado em meteorologia pela Universidade Federal de Campina Grande (UFCG), na qual obteve como resultado este trabalho.

\section{REFERÊNCIAS BIBLIOGRÁFICAS}

ALCÂNTARA, C. R.; SOUZA, E. P. Teoria para o trabalho de expansão aplicada às brisas do Nordeste Brasileiro. Revista Brasileira de Meteorologia, v. 24, p. 423-435, 2009.

COSTA, S. B. Balanços de vorticidade e energia aplicados aos Vórtices Ciclônicos de Altos Níveis atuantes no Oceano Atlântico Tropical Sul e adjacências. São Paulo, 2009. Dissertação (Mestrado em Meteorologia). USP, São Paulo, 2009.

EMANUEL, K. A. An air-sea interaction theory for tropical cyclones. Part I: Steady - State Maintenance. Journal of the Atmospheric Sciences, v. 43, p. 585 - 604, 1986.

FERREIRA, N. J.; CORREIA, A. A.; RAMIREZ, M. C. V. Synoptic scale features of the tropospheric circulation over tropical South America during the WETAMC TRMM/LBA experiment. Atmosféra [online], v. 17, n. 1, 2004.
FREITAS, S. R. et al. The Coupled Aerosol and Tracer Transport model to the Brazilian developments on the Regional Atmospheric Modeling System (CATT-BRAMS). Part 1: Model description and evaluation. Atmospheric Chemistry and Physics. Discuss, 7., 8525-8569, 2007.

GRELL, G. A.; DÉVÉNYI, D. A new approach to parameterizing convection using ensemble and data assimilation techniques. Geophysical Research Letters, v.29. Art. No p.1693, 2002.

KAROLY, D. J.; VICENT, D. G. American Meteorological Society, Meteorological Monographys, v. 27, n. 49, p. 119-139, 1998.

KAYANO, M. T.; ANDREOLI, R. V. Decadal variability of northern Brazil rainfall and its relation to tropical sea surface temperature and global sea level pressure anomalies. Journal of Geophysics Research, v. 109, n. C11011, DOI: 10.1029/2004JC002429, 2004.

KOUSKY, V. E.; GAN, M. A. Upper troposhperic cyclonic vortices in the tropical South Altantic. Tellus, v.33, p.538551, 1981.

LIMA, A. G. Contribuição de um sistema convectivo de mesoescala para o armazenamento de água na Paraíba. Campina Grande, 2009. Dissertação (Mestrado em Meteorologia). Universidade Federal de Campina Grande, Paraíba, 2009.

LORENZ, E. N. The nature and theory of the general circulation of the atmosphere. World Meteorological Organization, 161 pp., 1967.

MISHRA, S. K.; RAO, V. B.; GAN, M. A.. Structure and evolution of the large-scale flow of an embedded uppertropospheric cyclonic vortex over Northeast Brazil. Monthly Weather Review, v. 129, p. 1673-1688, 2001.

NEWELL, R. E. et al.. The general circulation of the tropical atmosphere and interactions with extratropical latitudes. Massachusets Institute of Technology Press, v. 1, 258 pp. 1972.

RAMIREZ, M. C. V.; FERREIRA, N. J.; KAYANO, M. T. Statistical analysis of upper tropospheric vórtices in the vicinities of northeast Brazil during the 1980-1989 period. Atmosféra (México), v. 12, p. 75-88, 1999.

RENNÓ, N. O. A thermodynamically general theory for convective vortices. Tellus, v. 60, p. $688-699,2008$. 
RENNÓ, N. O.; BLUESTEIN, H. B. A simple theory for waterspouts. Journal of the Atmospheric Sciences, v. 58, p. $927-932,2001$.

RENNÓ, N. O., et al. A simple theory for dust devils. Journal of the Atmospheric Sciences, v. 55, p. $3244-3252,1998$.

RENNÓ, N. O; INGERSOLL, A. P. Natural convection as a heat engine: A theory for CAPE. Journal of the Atmospheric Sciences, v. 53, n. 4, p. 572 - 585, 1996.

SATYAMURTY, P.; NOBRE, C. A.; SILVA DIAS, P. L. South America. In: Meteorology of the Southern Hemisphere. 1998.
SOUZA, E. P. Trabalho de expansão e de compressão associado às circulações convectivas. Revista Brasileira de Meteorologia, v. 19, n. 2, p. 141 - 148, 2004.

SOUZA, E. P.; RENNÓ, N. O. Convective circulations induced by surface heterogeneities. Journal of the Atmospheric Sciences, v. 57, p. 2915 - 2922, 2000.

WOOLARD, E. W. Recent investigations on the energy in the Earth's atmosphere, its transformation and dissipation. Monthly Weather Review, p. 254-255, 1926. 